\title{
The Netherlands protocol for standardisation and quantification of FDG whole body PET studies in multi-centre trials
}

\author{
Ronald Boellaard • Wim J. G. Oyen • \\ Corneline J. Hoekstra • Otto S. Hoekstra • \\ Eric P. Visser • Antoon T. Willemsen • Bertjan Arends • \\ Fred J. Verzijlbergen • Josee Zijlstra • Anne M. Paans • \\ Emile F. I. Comans • Jan Pruim
}

Received: 9 June 2008 / Accepted: 11 June 2008 / Published online: 15 August 2008

(C) The Author(s) 2008. This article is published with open access at Springerlink.com

\begin{abstract}
Introduction Several studies have shown the usefulness of positron emission tomography (PET) quantification using standardised uptake values (SUV) for diagnosis and staging, prognosis and response monitoring. Many factors affect SUV, such as patient preparation procedures, scan acquisition, image reconstruction and data analysis settings, and the variability in methodology across centres prohibits exchange of SUV data. Therefore, standardisation of 2-[ $\left.{ }^{18} \mathrm{~F}\right]$ fluoro-2-deoxy-D-glucose (FDG) PET whole body procedures is required in multi-centre trials.

Methods A protocol for standardisation of quantitative FDG whole body PET studies in the Netherlands (NL) was defined.
\end{abstract}

R. Boellaard $(\bowtie) \cdot$ O. S. Hoekstra $\cdot$ J. Zijlstra $・$ E. F. I. Comans Department of Nuclear Medicine \& PET Research,

VU University Medical Center,

De Boelelaan 1117, 1081 HV Amsterdam, The Netherlands

e-mail: r.boellaard@vumc.nl

W. J. G. Oyen • E. P. Visser

Department of Nuclear Medicine,

Radbound University Nijmegen Medical Center,

P.O. Box 9101, 6500 HB Nijmegen, The Netherlands

\section{J. Hoekstra}

Department of Nuclear Medicine, Jeroen Bosch Hospital, P.O. Box 90153, 5200 ME, 's-Hertogenbosch, The Netherlands

\section{A. T. Willemsen}

Department of Nuclear Medicine and Molecular Imaging,

University Medical Center Groningen,

Hanzeplein 1, 9700 RB Groningen, The Netherlands
This protocol is based on standardisation of: (1) patient preparation; (2) matching of scan statistics by prescribing dosage as function of patient weight, scan time per bed position, percentage of bed overlap and image acquisition mode (2D or 3D); (3) matching of image resolution by prescribing reconstruction settings for each type of scanner; (4) matching of data analysis procedure by defining volume of interest methods and SUV calculations and; (5) finally, a multi-centre QC procedure is defined using a 20-cm diameter phantom for verification of scanner calibration and the NEMA NU 22001 Image Quality phantom for verification of activity concentration recoveries (i.e., verification of image resolution and reconstruction convergence).

B. Arends

Department of Clinical Physics, Catharina Hospital, Michelangelolaan 2, 5623 EJ Eindhoven, The Netherlands

F. J. Verzijlbergen

Department of Nuclear Medicine, Sint Antonius Hospital, Koekoekslaan 1, 3435 CM Nieuwengein, The Netherlands

\footnotetext{
A. M. Paans $\cdot$ J. Pruim

Department of Nuclear Medicine and Molecular Imaging,

University Medical Center Groningen,

Hanzeplein 1, Postbus 30.001, 9700 RB Groningen,

The Netherlands
} 
Discussion This paper describes a protocol for standardization of quantitative FDG whole body multi-centre PET studies.

Conclusion The protocol was successfully implemented in the Netherlands and has been approved by the Netherlands Society of Nuclear Medicine.

Keywords Standardisation $\cdot$ FDG $\cdot$ PET .

Multi-centre trials $\cdot \mathrm{QC} \cdot \mathrm{SUV} \cdot$ Quantification

\section{Quantification of FDG whole body PET studies and need for standardisation of FDG-PET studies}

Positron emission tomography (PET) using 2- $\left[{ }^{18}\right.$ F $]$ fluoro-2deoxy-D-glucose (FDG) has become an important tool for diagnosis, staging, prognosis and treatment-response monitoring in oncology [1]. Vansteenkiste and Stroobants [2] reviewed the role of FDG-PET in respiratory oncology. In their paper, the authors addresses various aspects of FDG-PET in oncology and discuss amongst others that improved mediastinal lymph node staging of patients is obtained using PET compared with computed tomography(CT) alone. De GeusOei et al. [3] performed a systematic review on the predictive and prognostic value of FDG-PET in non-small cell lung cancer and concluded that the degree of FDG uptake is of prognostic value at initial presentation, after induction treatment before resection, and in case of recurrence. Weber et al. [4] studied the use of PET for monitoring cancer therapy and for predicting outcome. In his paper, the author addresses many issues involved in quantitative assessment of therapy-induced changes in FDG tumour uptake. To date, there are numerous papers on the promising role of FDG-PET in oncology [5-9].

While the important potential of FDG-PET in oncology has been demonstrated in many investigations and papers, many of these studies discuss the need for standardisation, especially for multi-centre trials and for meta-analyses. Standardisation becomes especially important when quantification of FDG-PET studies using standardised uptake values (SUV) is performed [10-12]. Although visual inspection of FDG-PET images remains very important for diagnosis and response assessment, it has been shown that semiquantitative analysis (SUV) allows an objective assessment for lesion characterisation [13], prognostic stratification [3] and monitoring treatment response [14]. The latter is generally measured by the relative change of SUV during treatment. At the same time, comparison of SUV results obtained from different centres is hampered by the disperse variability in methodology of acquisition, image reconstruction and data analysis procedures applied. Boellaard et al. [10] showed that many technical factors, such as image reconstruction settings and region of interest strategies, can have a major impact on the outcome of SUV. Jaskowiak et al. [15] showed the impact of
OSEM reconstruction settings on SUV. Stahl et al. [16] and Krak et al. [17] evaluated the effects of various clinical and technical factors on test-retest variability and absolute outcome of SUV and the impact of using various SUV measures on observed treatment response. Recently, Westerterp et al. [12] evaluated quantification of FDG-PET studies in multicentre trials with a focus on methodological variability among centres. These and other studies showed the need for harmonisation of FDG-PET across centres.

The need for standardisation was already addressed in 1999 by a position paper of Young et al. [18]. In that paper, various methods for quantification of FDG-PET studies, such as visual inspection, use of SUV or full kinetic analysis, were discussed. In addition, factors affecting FDG uptake are described. Taking into account these factors, a set of recommendations was presented. More recently, in 2006, Shankar et al. [19] published the concensus recommendations for FDG-PET studies as indicator of therapeutic response in patients in National Cancer Institute Trials. The guidelines given in the latter paper focus on patient preparation, image acquisition, image reconstruction, quantitative and semiquantitative image analysis, quality assurance, reproducibility and other factors in FDG-PET studies before and after a therapeutic intervention. Larson and Schwartz [20] stated that these NCI guidelines provide an excellent starting point for standardisation of the use of FDG-PET in clinical trials. Other authors, such as Zijlstra et al. [21] and Juweid et al. [22], stressed on the need for harmonisation of interpretation of FDG-PET studies in lymphoma either using visual inspection and/or using relative changes in SUV. More guidelines are being developed as technology moves forward [23]. Recently, Delbeke et al. [24] therefore provided procedure guidelines for tumour imaging with FDG PET-CT.

Although many issues are addressed in the aforementioned papers, there is still a need for standardisation of FDG-PET studies in case of multi-centre studies focussing on quantification using SUV. Therefore, a PET imaging working group was formed by the Dutch Society of Haemato-Oncology (HOVON, Hemato-Oncologie Vereniging voor Volwassenen in Nederland) and by Nuclear Medicine Physicians, representatives of the Dutch Society of Nuclear Medicine, and Medical Physicists, representatives of the Dutch Society of Nuclear Medicine and Dutch Society of Clinical Physics, with the task of formulating a protocol for standardisation and quantification of FDG Whole Body PET studies in multicentre trials in The Netherlands. The protocol specifically aims at standardisation of FDG-PET studies in order to allow inter-institute interchangeability of SUV. The first version of the protocol was finalized in November 2007 and was approved by the Dutch Society of Nuclear Medicine. The purpose of the present paper is to provide a description of the protocol and the rationale for recommendations given. First, factors affecting SUV outcome will be summarized shortly as 
these have been addressed extensively elsewhere. Next, guidelines and recommendations will be described in the main part of the paper. Finally, limitations, pending issues and future work will be addressed.

\section{A brief summary of factors affecting SUV}

Various factors and sources of error affect SUV outcome. These factors can be assigned to physiological effects, sources of error and physical effects.

Physiological factors affecting SUV are, for example, the plasma glucose level during FDG-PET scanning, FDG plasma clearance during the phase of FDG uptake, the scanning period and the effects of patient motion and breathing. As FDG uptake is time dependent, the time interval applied between FDG administration and execution of the PET study will affect SUV as well. For response monitoring, the time interval between the baseline FDG-PET study and start of treatment as well as the time intervals between subsequent FDG-PET studies and cycles of treatment are of importance. This is especially important when inflammatory processes may occur as may be the case during or after radiation therapy. These factors and their impact on SUV outcome are summarized in Table 1.

Table 2 presents some of the most common errors affecting SUV, as also described by Weber et al. [4] and Shankar et al. [19]. Correct calculation of SUV depends on an accurate (cross-)calibration between PET or PET-CT scanner and the dose calibrator used for measuring patient dose. Moreover, the net dose given to the patient needs to be known exactly.

Table 1 Biological factor affecting SUV outcome

\begin{tabular}{|c|c|}
\hline Biological factor & Effect \\
\hline Blood glucose level & $\begin{array}{l}\text { Lower uptake or SUV with increasing } \\
\text { blood glucose level }\end{array}$ \\
\hline Uptake period & $\begin{array}{l}\text { Higher SUV at increasing time } \\
\text { interval between injection and start } \\
\text { of PET study }\end{array}$ \\
\hline Patient motion/breathing & $\begin{array}{l}\text { Image artefacts in case of mismatch in } \\
\text { position between CT-AC and emission } \\
\text { scan and (possibly) lower SUV due to } \\
\text { respiratory motion (resolution loss) }\end{array}$ \\
\hline Patient comfort & $\begin{array}{l}\text { Patient stress and uncomfortable waiting } \\
\text { conditions increase uptake of FDG in } \\
\text { muscle and/or brown fat and may affect } \\
\text { SUV quantification, also in case of low } \\
\text { tumour-to-background ratios due to } \\
\text { spillover. }\end{array}$ \\
\hline Inflammation & $\begin{array}{l}\text { Inflammatory processes near or at the } \\
\text { tumour results in a false positive } \\
\text { increase of SUV. }\end{array}$ \\
\hline
\end{tabular}

Thus, any remaining activity in the syringe after administration should be minimized to within $1 \%$ using appropriate procedures. Alternatively, remaining activity in the syringe may be measured after the administration. In the majority $(95 \%)$ of the cases, remaining activity in the syringe is smaller than $3 \%$ of the specified dose, although occasionally ( $5 \%$ of the cases) remaining activity was more than $10 \%$ (unpublished data), which mainly occurred when specific activities are still high (i.e., shortly after production). The effects of dead volume in the syringe preparation system and administration system should thus be carefully taken into account. A subtraction method to derive the amount of activity in a syringe may be used. The latter is generally based on subtracting a small amount of FDG from a stock, positioned in a dose calibrator, and measuring the amount of activity of the stock prior and after withdrawal of the patient dose. If the stock contains a large amount of activity relative to that needed for the patient dose, care has to be taken that dead volume or statistical errors do not affect accuracy and reproducibility of net dose assessment. With proper procedures, however, this can be handled easily. A frequently overlooked problem may arise from an incorrect synchronisation of clocks in a PET system with those of the dose calibrator used (or computer attached to it for read-out and/or) or with clocks in various rooms/locations. Moreover, as the patient dose is specified at a certain dose calibration time, which is generally not exactly equal to the actual injection time, correct decay corrections have to be applied. This means that decay between calibration time and PET acquisition time needs to be applied to the dose specified at the dose calibration time. Obviously, paravenous injection may still allow visual interpretation of the PET study, but will make quantification based on SUV incorrect. A reproducible and correct administration of FDG is required.

Physical or technical factors affecting SUV outcome (Table 3) are described in various papers. Data acquisition settings such as time per bed position, the amount of overlap between subsequent bed positions, acquisition mode (2D or 3D) and FDG dose affect scan statistics and/or the noise equivalent count level of PET studies. Poorer scan statistics and lower image signal-to-noise ratio (SNR) will result in an upward bias of SUV, especially when using the maximum SUV as final outcome parameter [10].

Nowadays, attenuation corrections will be mostly derived from a dedicated CT scan (CT-AC) acquired on a PET-CT scanner. Patient motion (e.g., breathing) may result in a mismatch between PET and CT and thus result in incorrect attenuation correction. The latter may be minimized by breathing instructions (breath holding at mid-inspiration volume or shallow breathing) during $\mathrm{CT}$ scanning. Use of respiratory gating would be beneficial, but is not yet routinely available in most centres. Use of contrast agents may affect accuracy of conversion of CT-AC image Hounsfield units into $511 \mathrm{keV}$ 
Table 2 Errors affecting SUV

\begin{tabular}{|c|c|}
\hline Error & Effect \\
\hline Cross-calibration between PET and dose calibrator (DC) & $\begin{array}{l}\text { Systematic error in SUV equal to error in (relative) cross-calibration } \\
\text { between PET and DC }\end{array}$ \\
\hline Residual activities in administration system (syringe, lines etc.) & Lower net administered dose resulting in incorrect lower uptake and SUV \\
\hline Incorrect synchronisation of clocks of PET camera and DC & Incorrect decay correction resulting in incorrect SUV \\
\hline $\begin{array}{l}\text { Use of injection time rather than dose calibration time (or use of } \\
\text { incorrect amount of dose) }\end{array}$ & $\begin{array}{l}\text { Incorrect time interval is used for decay correction of administered dose } \\
\text { resulting in incorrect SUV }\end{array}$ \\
\hline Paravenous administration of FDG & $\begin{array}{l}\text { Paravenous injection results in slow delivery of FDG to the tumour and } \\
\text { therefore in incorrect SUV }\end{array}$ \\
\hline
\end{tabular}

linear attenuation correction factors and thereby accuracy of SUV. Hopefully, sophisticated algorithms to perform this conversion will be introduced allowing for use of contrast agents, as will be discussed in more detail later. Finally, attenuation correction based on CT could be affected by truncation of the CT image. Note that CT truncation does not only affect the quantification of PET at the position of CT image truncation alone, but may affect SUV of tumours located elsewhere in the same axial planes as well.

Image reconstruction methods and settings determine both quantitative accuracy and final image resolution. With iterative reconstruction methods, a sufficient amount of iterations (or the product of the number of iterations and subsets) need to be applied to ensure sufficient convergence of the algorithm. Insufficient convergence will result in an object- (and/or surroundings) dependent SUV outcome [15]. In addition to these settings, generally some filtering is performed during or post-reconstruction. Typically, Gaussian filters of 5 to $10 \mathrm{~mm}$ full width at half maximum (FWHM) are applied resulting in a clinical image resolution of 7 to $12 \mathrm{~mm}$ FWHM. Image matrix size and zoom factors applied during reconstruction determine the final voxel size and may lead to additional image resolution loss when voxel size is larger than half the intended FWHM (Nyquist criteria). The final image resolution has a large effect on so-called partial volume effects
(PVE), which results in an underestimation of SUV for small objects. PVE increases with decreasing image resolution. PVE becomes mainly important for lesions smaller than three times the FWHM. To date, no accurate and precise PVE correction are available for objects smaller than about two times the FWHM (i.e., generally $<15$ to $20 \mathrm{~mm}$ diameter). Consequently, image resolution must be matched as closely as possible among centres in multi-centre trials to avoid differences in SUV due to difference in image resolution. Other data acquisition setting and reconstruction parameters, such as the use of time of flight and relaxation parameters, affect image quality and SUV outcome. When images have been collected, additional variability in SUV results from different data analysis strategies, especially region of interest (ROI) definitions. Fixed size ROI providing 'SUV peak', maximum pixel values providing 'SUVmax' or the average ROI value within a 2D or 3D ROI providing 'SUV mean' are being used. Moreover, 2D and 3D ROI can be generated manually or using various semiautomatic procedures, the latter generally based on some percentage threshold value relative to maximum SUV. Examples of the effects of the number of OSEM iterations and different image resolutions using various ROI on SUV are shown in Fig. 1. Finally, various SUV normalisations are being utilized such as SUV normalised by body weight, body surface area or lean body mass.

Table 3 Technical factors affecting SUV

\begin{tabular}{ll}
\hline Factor & Effect \\
\hline $\begin{array}{l}\text { Scan acquisition parameters (acquisition mode, scan } \\
\text { duration, bed overlap, FDG dose) }\end{array}$ & $\begin{array}{c}\text { Affect signal to noise ratio (SNR) of PET scan. Poorer SNR results in an upward bias of } \\
\text { SUV. }\end{array}$ \\
$\begin{array}{l}\text { Image reconstruction settings (number of iterations, } \\
\text { filters, matrix size, zoom factors) }\end{array}$ & $\begin{array}{c}\text { Affect convergence of iterative reconstructions and final spatial resolution (filter, matrix, } \\
\text { zoom) of reconstructed image. Insufficient convergence and lower resolution results in } \\
\text { lower SUV and increases partial volume effects. Moreover, insufficient convergence } \\
\text { makes SUV more dependent on surrounding activity distributions. }\end{array}$ \\
$\begin{array}{l}\text { Hegion of interest (ROI) strategy to derive SUV lower SUV depending on size and type of ROI used. } \\
\text { Normalisation factor in SUV calculation }\end{array}$ & $\begin{array}{c}\text { SUV outcomes are numerically different when using body weight, body surface area or } \\
\text { lean body mass as normalisation factor in the SUV equation }\end{array}$ \\
$\begin{array}{l}\text { Higher blood glucose levels will result in underestimation of SUV. Use of a blood } \\
\text { glucose level correction in the SUV equation will thus result in different SUV outcomes. }\end{array}$ \\
$\begin{array}{c}\text { Can result in overestimation of attenuation and thus results in higher SUV (upward bias). } \\
\text { Use of contrast agents during CT-AC }\end{array}$
\end{tabular}




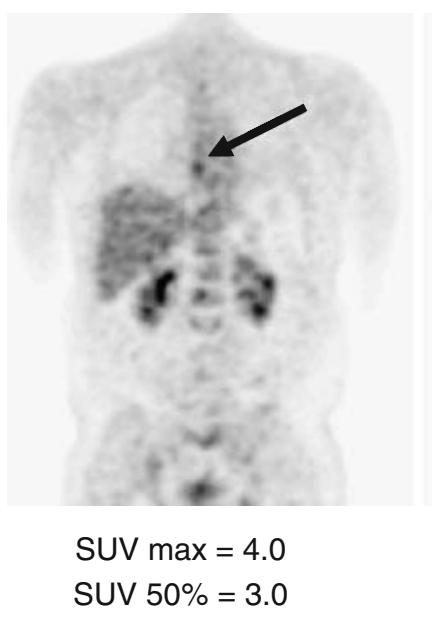

(A)

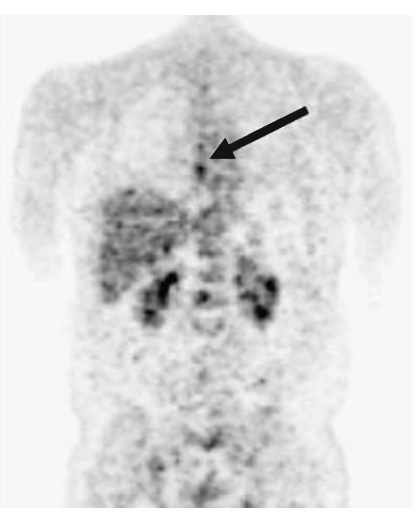

5.9

4.1

(B)

Fig. 1 Images illustrating effects of the number of OSEM iterations and image smoothing on SUV. Representative image reconstruction settings, as observed in a previous multicentre study, were taken. Images were reconstructed using OSEM with two iterations, eight

\section{A standardised protocol for FDG-PET whole body studies}

The protocol for standardisation and quantification of FDGPET whole body studies is based on principles for minimizing intersubject and inter-institute variability of SUV measures:

- Recommendations for patient preparation are specified in order to minimize patient related or other physiological effects on SUV accuracy and reproducibility. Moreover, guidelines aim at optimizing FDG uptake in the tumour, minimizing uptake in surrounding tissues (muscle, brown fat) and minimizing SUV variability.

- Recommendations for the administration procedure are given to ensure that the net dose given to the patient is exactly known by avoiding (unknown) remaining activities during preparation and administration.

- FDG dose is specified as a function of patient weight, scanning mode, percentage bed overlap, and acquisition time per bed position. In this way, it is attempted to obtain a smaller range in scan statistics between subjects, because of a difference in weight, and between different scanners, due to difference in sensitivity and applied overlap of subsequent scan bed positions.

- Interchangeability of SUV (or it's absence) is, to a large extent, affected by the overall spatial resolution of the PET images after reconstruction, filtering or smoothing and processing. Resolution matching across scanners or centres is therefore needed to allow for SUV interchangeability.

- After PET acquisition and image reconstruction, SUV outcome is determined by data analysis procedures. Common region of interest (ROI) strategies are the use of fixed sized 2D or 3D ROI, manually defined ROI in one or more axial slices and 3D ROI based on region growing procedures while applying a user-specified threshold. Preferred and obligatory ROI therefore need to be specified.

- Various SUV normalisations can be applied such as body weight, body surface area and lean body mass. Moreover, SUV may be corrected for blood glucose level. In order to interpret reported SUV it is mandatory to clearly indicate and specify which SUV measure will be used.

- Finally, it is essential that (1) the PET scanner is technically operating according to specifications, that (2) the PET scanner and the dose calibrator used to determine patient FDG dose are calibrated against each other correctly, including synchronisation of clocks used within the department, and that (3) performance of PET scanner and the characteristics of reconstructed PET studies, such as SNR, uniformity and spatial resolution (specified in terms of activity recovery coefficients) remain consistent or are known after hardware, firmware and software upgrades. To this end, multicentre $Q C$ measures are therefore specified.

Patient preparation

1. Patients should have fasted for at least $4 \mathrm{~h}$ and preferably $6 \mathrm{~h}$ before administration of FDG.

2. Ample hydration is recommended by intake of 11 of water during $2 \mathrm{~h}$ prior to administration of FDG. In case of venous hydration, no glucose may be present in the infusate.

3. After administration of FDG, patients should drink 0.51 water before the PET examination. When necessary, 0.51 water or saline can be given intraveneously. 
4. No or minimal exercise $4 \mathrm{~h}$ prior to and after FDG administration and during the entire PET examination. However, patients are allowed to visit the rest room. Instruction should be given to the patients to relax, avoid motion and unnecessary talking.

5. Patients are asked to void the bladder shortly (5 to $10 \mathrm{~min}$ ) before the PET examination.

6. Blood glucose level must be measured before administration of FDG. If blood glucose level is greater than $11 \mathrm{mmol} / \mathrm{l}$, the patient must be rescheduled. In case blood glucose level is being used to correct SUV, it must be measured at the clinical laboratory of the hospital using valid methodology.

7. Waiting conditions, preparation room and room temperature must be comfortable to allow optimal resting conditions during and after FDG administration in order to minimize muscle and brown-fat uptake. Blankets must be provided to the patient when needed/requested.

8. An urinary catheter may be used upon indication, preferably placed before administration of FDG.

9. Administration of a diuretic may be considered in case of expected pathology in the lower abdomen.

10. Routine use of tranquilizers (benzodiazepines) is not recommended, but may be considered in case tumours are expected in the head and neck region to minimize FDG muscle uptake. Benzodiazepines have no further use for suppressing the so-called brown fat phenomenon.

11. Patient weight and height must be measured using calibrated and medically approved devices.

12. Date of last cycle of chemotherapy, administration of growth factors (G-CSF, GM-CSF) or other treatments must be reported. Intervals between interventions and PET should be specified for each (research) protocol. For routine clinical care in solid tumours, an interval between end of last (chemo-)therapy cycle and FDG-PET must be at least 14 days. In case of radiation treatment, an interval up to 4 months may be required occasionally (e.g., larynx carcinoma). For malignant lymphoma therapy evaluation, time intervals have been specified by the Imaging Subcommittee of International Harmonization Project in Lymphoma [22].

In case of type II diabetes mellitus, the following additional guidelines apply:

1. Study will be scheduled preferably at the end of morning

2. Patients will need to be fasted for at least $4 \mathrm{~h}$. Intake of water is recommended as is nondiabetics.

3. Oral antidiabetic drugs should be continued.

In case of type I and insulin-dependent type II diabetes mellitus, the following additional guidelines apply:

1. It is preferred to reach normal blood glucose levels in mutual agreement with patient and referring physician
2. Study will be scheduled preferably at the end of morning

3. Patient will have a normal breakfast at 7:00 A.M. and uses a regular dose of insulin, followed by fasting as described above.

FDG administration procedure

The procedure aims at an accurate and reproducible administration of FDG, minimizing or avoiding remaining activity in the administration system and thus ensuring that the exact net dose administered is known. This is a prerequisite for accurate SUV calculations as discussed previously in the "A brief summary of factors affecting SUV" section because the net dose administered to the patient is used directly in the SUV equation (Eqs. 1 and 2).

It is preferred to administer FDG through a three-way valve system attached to a venous canula. After injection, the entire administration system should be flushed with at least $10 \mathrm{cc}$ saline to avoid remaining activity in the system. One should be aware of any activity remaining in the syringe, needle or administration system. If rest-activity in syringe, needle or administration system cannot be minimized using appropriate procedures $(<1 \%)$; remaining activity should be measured afterwards in a calibrated dose calibrator.

FDG dosage in relation to scanner type, patient weight and time per bed position

A prescription of FDG dosage as function of patient weight, scanner type, percentage bed overlap, and time per bed position is given to minimize variability in scan statistics across scanners and patients (with different weights). As an upward bias (maximum), SUV was seen with decreasing image SNR minimizing (referentie?), scan statistics variability across centres, and patients will thus improve interchangeability of SUV in multi-centre studies.

In principle, a dosage of $5 \mathrm{MBq} / \mathrm{kg}$ for $2 \mathrm{D}$ scanners and 2.5 MB/kg for 3D scanners with minimal bed overlap $(<25 \%)$ assuming a (fixed) $5 \mathrm{~min}$ emission scan per bed position is used. In case of 3D scanners with a $50 \%$ bed overlap of subsequent bed positions of a whole body PET scan $1.25 \mathrm{MBq} / \mathrm{kg}$ is sufficient. For shorter scan times per bed position, the dosage can be adjusted proportionally but with an additional surplus of $10 \%$ to (partly) compensate for lower noise equivalent count rates per $\mathrm{MBq}$ at higher doses. The following formulas apply to determine the patient specific FDG dosage (MBq):

1. For 2 D PET scanners with less than $25 \%$ bed overlap the total amount of FDG dose is given by:

a. Dosage $=27.5$ times patient weight $(\mathrm{kg})$ and divided by the scan time per bed position (min per bed) 
2. For 3D PET scanners with less than $25 \%$ bed overlap the total amount of FDG dose is given by:

a. Dosage $=13.8$ times patient weight $(\mathrm{kg})$ and divided by the scan time per bed position (min per bed)

3. For 3D PET scanners with $50 \%$ bed overlap the total amount of FDG dose is given by:

a. Dosage $=6.9$ times patient weight $(\mathrm{kg})$ and divided by the scan time per bed position (min per bed)

The following additional recommendations apply:

1. Net administered dose should be within $10 \%$ of the recommended dose but must be exactly known within $3 \%$.

2. Increasing the FDG dosage to reduce overall scanning time should not result in count rates exceeding the maximum count rate capabilities of the PET scanner, i.e., above which corrections for dead time or others can no longer be accurately performed $(<5 \%)$. When specified, the recommendations of the manufacturer on maximum dosage should be followed.

3. When variable scan times per bed position can be applied, it is allowed to reduce the scan time per bed position with $50 \%$ for scan trajectories outside thorax and abdomen (thus for head, neck and legs) to reduce overall scanning time. For the calculation of the FDG dose, it is still required to use the scan time per bed as needed for scans at the thorax and abdomen regions.

4. To facilitate clinical use, a table specifying required FDG dose in $10 \mathrm{~kg}$ patient weight steps may be used. An example of such a table is given in Table 4.

\section{Exceptions}

For new scanners, e.g., scanners with time of flight (ToF) or equipped with new reconstruction methods (e.g., recovery correction), it is allowed to deviate from the above specifications. In the latter case, it is up to the users to demonstrate that use of other than the recommended dose-scan time per bed specifications does not result in a change of observed SUV. To that end, the deviation from the above-given specifications must be justified using phantom experiments, as described later in the QC section of this protocol, as well as by a clinical study relevant to the ongoing multi-centre study (i.e., using the same patient population). In this way, this protocol facilitates a proper validation of new technology for quantitative FDGPET studies and ensures exchange of SUV in multi-centre studies while allowing benefits of new technology.

\section{PET and PET-CT acquisitions}

1. Emission scanning should start at 55 to $65 \mathrm{~min}$ after FDG administration

2. For most oncological applications, a 'whole body' scan trajectory extends from proximal thigh up to skull-base. Scanning direction should be in the caudocranial direction to have minimal bladder filling at the time of scanning the lower abdomen ('feet first'). Alternative scan trajectories can be used upon indication.

3. During PET-CT, arms should preferably be elevated over the head to minimize streak artefacts (in case of positioning along the body) due to, e.g., beam hardening of CT-AC.

4. Correct isotope and decay times should be set or entered in the acquisition computer.

Table 4 FDG dose as function of patient weight (10 kg steps)

\begin{tabular}{|c|c|c|c|c|c|c|c|c|c|}
\hline \multirow{4}{*}{$\begin{array}{l}\text { Mode } \\
\text { Time/bed } \\
\text { Bed overlap (\%) } \\
\text { Patient weight }(\mathrm{kg})\end{array}$} & $2 \mathrm{D}$ & $3 \mathrm{D}$ & $3 \mathrm{D}$ & $3 \mathrm{D}$ & $3 \mathrm{D}$ & $3 \mathrm{D}$ & $3 \mathrm{D}$ & $3 \mathrm{D}$ & $3 \mathrm{D}$ \\
\hline & 5 & 5 & 4 & 3 & 2 & 5 & 4 & 3 & 2 \\
\hline & 25 & 25 & 25 & 25 & 25 & 50 & 50 & 50 & 50 \\
\hline & \multicolumn{9}{|c|}{ FDG Dose (MBq) } \\
\hline $30-39$ & 170 & 80 & 120 & 160 & 240 & 40 & 60 & 80 & 120 \\
\hline $40-49$ & 220 & 110 & 150 & 200 & 310 & 60 & 70 & 100 & 150 \\
\hline $50-59$ & 270 & 130 & 180 & 250 & 370 & 70 & 90 & 120 & 180 \\
\hline $60-69$ & 320 & 160 & 220 & 290 & 440 & 80 & 110 & 140 & 220 \\
\hline $70-79$ & 370 & 180 & 250 & 340 & 510 & 100 & 120 & 170 & 250 \\
\hline $80-89$ & 420 & 210 & 290 & 390 & 580 & 110 & 140 & 190 & 290 \\
\hline $90-99$ & 470 & 230 & 320 & 430 & 650 & 130 & 160 & 210 & 320 \\
\hline $100-109$ & 520 & 260 & 360 & 480 & 720 & 140 & 180 & 240 & 360 \\
\hline 110-119 & 570 & 280 & 390 & 520 & - & 150 & 190 & 260 & 390 \\
\hline $120-129$ & 620 & 310 & 430 & 570 & - & 170 & 210 & 280 & 430 \\
\hline $130-139$ & 670 & 330 & 460 & 620 & - & 180 & 230 & 310 & 460 \\
\hline $140-149$ & 720 & 360 & 500 & 660 & - & 200 & 250 & 330 & 500 \\
\hline
\end{tabular}


5. FDG dosage, dose calibration time, patient weight must be correctly set when required by the acquisition software.

6. Online random correction based on delayed coincidence window technique is recommended.

7. For CT-based attenuation correction generally a low-dose CT is performed (CT-AC). For the CT-AC scan, the recommendations provided by the manufacturer may be followed. Generally, CT-AC scans are operated using $30 \mathrm{mAs}$ or less. During and prior to CT-AC scanning, no intravenous contrast agents may be used (until it has been unequivocally demonstrated that this does not affect SUV).

8. During CT-AC, shallow breathing is recommended for multi-slice CT scanners to minimize effects of patient motion on attenuation correction.

9. General PET-CT procedure consists of a scout scan for positioning the PET and CT scan trajectories. Next, a low-dose CT scan for attenuation correction is performed followed by the emission scan. Upon indication, the procedure may be concluded by a diagnostic CT scan including use of intravenous contrast agents and applying maximal inspiration breath hold. Diagnostic CT settings should be according to specifications provided by the radiologist and/ or specialised medical physicist.

Pitfalls and additional recommendations:

1. For certain PET-CT scanners, the CT or CT-AC FOV may be smaller than that of the PET. Therefore, truncation of CT-AC should be avoided to avoid attenuation (and scatter) correction artefacts and quantification errors.

2. In case transmission scans based on radioactive sources (generally Ge-68 line sources) are used for attenuation correction, these sources must have sufficient strength to acquire transmission scans. Low transmission source activity will result in poor SNR in transmission scans affecting image quality and quantification of emission scans. Transmission scan sources should fulfill criteria specified by the manufacturer. Moreover, Ge-68 line sources need to be replaced at least every 1.5 years.
PET image reconstruction

Based on a phantom study (Fig. 2), it was observed that most modern PET-CT scanners provide images with a spatial resolution close to $7 \mathrm{~mm}$ FWHM when using the default by the manufacturer-recommended image reconstruction settings. It is therefore attempted that PET studies from various scanners and centres will have a final image resolution as close as possible to $7 \mathrm{~mm}$ FWHM. When possible, specific image reconstruction algorithms and settings are defined per scanner. Settings are chosen such that, for iterative reconstructions, sufficient convergence is achieved. Other settings, such as smoothing filters and image matrix size, will be chosen such that final image resolution will be close to $7 \mathrm{~mm}$ FWHM. Optimal settings need to be derived so that activity concentration recovery coefficients as function of sphere size will meet QC specifications, as described later (Fig. 2). The latter procedure is followed, as use of point sources for verification of image resolution should not be used in case of iterative reconstruction methods, which are exclusively used nowadays for FDG-PET whole body studies. Moreover, variably sized spheres, simulating tumours, resemble clinical conditions more closely than point sources. The phantom experiments, as part of multicentre QC procedures, will be described later.

The following reconstruction parameters are defined.

1. All correction methods needed for quantification need to be applied. These include decay correction, dead time correction, corrections for detector efficiencies (normalisation), random correction, attenuation correction and model-based scatter correction, such as the single scatter simulation method by Watson et al. [25] or similar ones

2. Additional and indicative reconstruction settings are suggested.

For Siemens/CTI scanners:

- (FORE+) 2D OSEM reconstruction with four iterations and 16 subsets.

- A post reconstruction smoothing using a 5-mm FWHM Gaussian filter
Fig. 2 Average ( $\pm 1 \mathrm{SD})$ activity concentration recovery coefficients as function of sphere size observed with image quality control measurements at eight different scanners using a an adaptive $50 \%$ isocountour VOI (A50\%) and b maximum pixel value

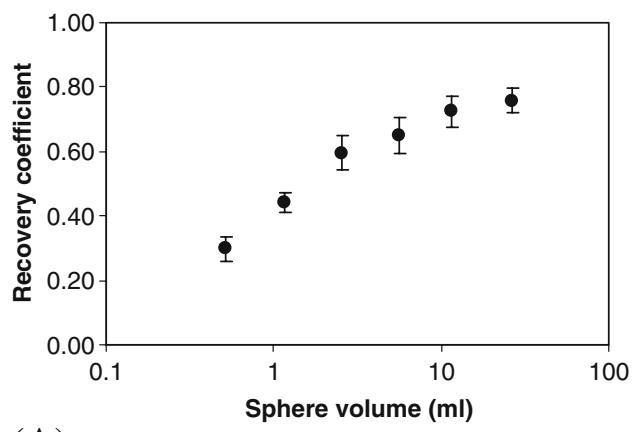

(A)

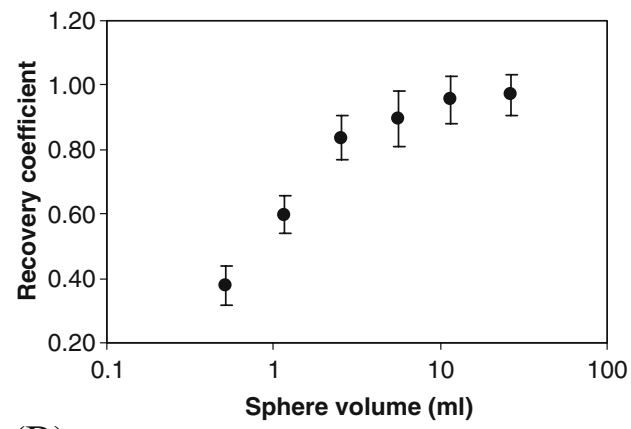

(B) 
- Model-based scatter correction.

- Matrix size of $128 \times 128$

- Zoom $=1.0$

For GE scanners:

- Default reconstruction settings (generally OSEM with about two iterations and 30 subsets for 2D scans and FORE+2D OSEM with five iterations and 21 subsets for $3 \mathrm{D}$ scans)

- A post-reconstruction smoothing using a 5-mm FWHM Gaussian filter

- Model-based scatter correction

- Matrix size of $128 \times 128$

- Zoom $=1.0$

For Philips scanners:

- For scanners equipped with time of flight (ToF), the default reconstruction methods (LOR based reconstruction including ToF) should be used applying 'normal' smoothing setting

- For scanners without ToF, reconstructions should be performed using LOR-RAMLA or 3D RAMLA applying four iterations and other settings as specified by the manufacturer.

- Matrix size of $144 \times 144$ (default)

- Zoom $=1.0$

\section{Exceptions and additional remarks}

As technology moves forward and new scanners and/or new reconstruction algorithms are being developed, the aforementioned scanner-specific recommendations may become obsolete. Consequently, it is allowed to deviate from the recommended reconstruction parameters provided that reconstructed images still meet the quality control criteria, especially those for activity concentration recovery coefficients measured in the NEMA NU 22001 Image Quality phantom, as specified later in the QC section.

Scanners which are not equipped with accurate random correction (based on singles or delayed coincidence time window technique) or with model-based scatter correction cannot be used when absolute SUV quantification is needed. However, these scanners may still be used in studies in which relative SUV changes are used to assess treatment response provided that the patient is scanned in an identical manner on the same scanner and using the same reconstruction settings and analysis methods during all subsequent scans.

Data analysis — volume of interest definitions

Beyond acquisition and image reconstruction, the SUV result is determined by data analysis procedures. Common region or volume of interest (ROI, VOI) strategies are the use of fixedsized 2D or 3D ROI, manually defined ROI in one or more axial slices and 3D ROI based on region-growing procedures while applying a user-specified threshold. In order to exchange SUV across centres, referred and obligatory ROI need to be specified.

The following regions of interest are suggested to be useful

- 3D isocount contour at $41 \%$ of maximum uptake including local background corrections, i.e., an adaptive $41 \%$ VOI $\left(\mathrm{VOI}_{\mathrm{A} 41}\right)[10,26]$.

- $3 \mathrm{D}$ isocount contour at $50 \%$ of maximum uptake $\left(\mathrm{VOI}_{50}\right)$

- 3D isocount contour at $50 \%$ of maximum uptake including local background corrections $\left(\mathrm{VOI}_{\mathrm{A} 50}\right)[10,17]$.

- 3D isocount contour at $70 \%$ of maximum uptake $\left(\mathrm{VOI}_{70}\right)$.

- 3D isocount contour at 70\% of maximum uptake including local background corrections $\left(\mathrm{VOI}_{\mathrm{A} 70}\right)[10,17]$.

- Maximum voxel value (Max)

Use of $\mathrm{VOI}_{\mathrm{A} 41}$ is based on results of recovery coefficient phantom experiments, such as the one described in the QC section later on, in which it can observed that using an adaptive $41 \%$ threshold provides VOI with a volume close to real sphere volumes used. Moreover, this or a similar threshold VOI has been suggested for radiation oncology purposes [26-29], although many other VOI strategies have been suggested for the latter purpose as well $[30,31]$.

Use of $\mathrm{VOI}_{50}, \mathrm{VOI}_{\mathrm{A} 50}, \mathrm{VOI}_{70}, \mathrm{VOI}_{\mathrm{A} 70}$ are commonly applied VOI and are included to allow comparison of SUV with those reported in previous studies. The maximum pixel value is nowadays most frequently used, and it is especially attractive as it does not require specialized algorithms to automatically generate VOI, and it does not suffer from observer variability as may be seen when using manually defined VOI.

VOI recommendations:

1. The maximum voxel value or maximum $\mathrm{SUV}\left(\mathrm{SUV}_{\max }\right)$ must always be reported.

2. Use of larger VOI may provide SUV estimates with better precision (smaller uncertainty). Therefore, in a response monitoring settings, use of $\mathrm{VOI}_{\mathrm{A} 41}$ should be attempted in all sequential scans of a patient. When $\mathrm{VOI}_{\mathrm{A} 41}$ does not provide meaningful tumour definitions, as needs to be checked visually, smaller VOI obtained with higher percentage threshold values can be applied $[10,12,17]$. Subsequently and in this order, $\mathrm{VOI}_{50}, \mathrm{VOI}_{\mathrm{A} 50}, \mathrm{VOI}_{70}$, $\mathrm{VOI}_{\mathrm{A} 70}$ may then be used consistently in all scans of a subject. However, when it is expected that one or more of these VOI may not be useful for certain studies, it is optional to agree upon the use of $\mathrm{VOI}_{70}$ and the maximum uptake beforehand. In all cases, $\mathrm{SUV}_{\max }$ and its 
change need to be reported in addition to all other VOI strategies agreed upon.

3. Automatic procedures for generating 3D isocount contours may fail in case of low tumour-to-background ratios or in the vicinity of organs showing high uptake (bladder, heart, brain). It is recommended to visually check if the automatically generated volume of interest corresponds with the localisation of the lesion, i.e., without inclusion of non-tumour structures or organs. In case automated VOI strategies cannot be applied reliably, the maximum SUV should be derived from a manually drawn 3D volume of interest which includes the entire tumour and excludes normal tissue.

Data analysis - scan report and SUV calculation

1. PET and PET-CT scans will be evaluated visually directly from a computer screen by a trained Nuclear Medicine Physician. Attention will be paid to the presence or absence of abnormal focal FDG avidities as well as their size and uptake. Both images reconstructed with and without attenuation correction need to be evaluated to rule out artefacts due to patient motion, metal implants, or contrast agents. Findings need to be reported and correlated to those seen with other diagnostic modalities or findings (if any).

2. It is recommended to report SUV normalised by body weight. However, besides weight, also patient height and gender should be reported thereby allowing calculating other SUV measures. Use of blood glucose level correction is not mandatory, but may be performed in addition to non-blood glucose level corrected SUV measures. SUV normalised by body weight without or with blood glucose level correction are calculated as follows:

$$
\begin{aligned}
& \mathrm{SUV}=\frac{\mathrm{ACvoi}(\mathrm{kBq} / \mathrm{ml})}{\operatorname{FDGdose}(\mathrm{MBq}) / \mathrm{BW}(\mathrm{kg})} \\
& \mathrm{SUVglu}=\frac{\mathrm{ACvoi}(\mathrm{kBq} / \mathrm{ml})}{\operatorname{FDGdose}(\mathrm{MBq}) / \mathrm{BW}(\mathrm{kg})} \times \frac{\operatorname{Pglu}(\mathrm{mmol} / \mathrm{l})}{5.0}
\end{aligned}
$$

In Eqs. 1 and 2, ACvoi represent the average activity concentration in $\mathrm{kBq} / \mathrm{ml}$ in the specified volume of interest (or the maximum value). FDGdose is the administered dose of FDG in $\mathrm{MBq}$ and $\mathrm{BW}$ is the body weight in $\mathrm{kg}$. Pglu represent blood glucose level in $\mathrm{mmol} / \mathrm{l}$. In case SUV is corrected for blood glucose level, i.e., SUVglu, the observed
Pglu is normalised by 5.0 representing approximately the population average Pglu value.

\section{Data exchange}

When data (FDG-PET images) need to be exchanged within a multi-centre study, it is required to specify image data format and meta-data required for accurate SUV calculations. DICOM images may be stored in various units, such as 'counts', 'SUV' or "Bq/cc". Therefore, when using DICOM format, one has to verify that all factors (scaling and calibration factors) are present in the header of the DICOM files in order to allow quantification of the images.

The following (minimal) recommendations are specified:

DICOM format should meet the specifications "Digital Imaging and Communications in Medicine (DICOM), Part 10: Media Storage and File Format for Media Interchange "given by National Electrical Manufacturers Association (NEMA; http://dicom.nema.org/)

In addition, DICOM format should meet the Conformance Statement written by the manufacturer of the PET or PET-CT scanner.

All data, including DICOM images, need to be anonymised. Patient name and ID need to be erased or replace by study ID and randomisation number, respectively, before sending these data to other centres. The birth date needs to be erased as well. This is especially required when data are exchanged over the Internet.

DICOM images or headers have to contain all factors (scaling and calibration factors) required for quantification of the images.

Transport of data or DICOM images may be performed by storing it on media (CD or DVD) and sending it by regular mail to other centres. However, electronic transport using $\mathrm{ftp}$ is allowed provided that $\mathrm{ftp}$ is secured and ftp-server password protected. Moreover, all (image) data need to be anonymised.

All additional information needed to calculate SUV must be provided. This information, such as study ID, patient randomnisation number, patient weight, height, gender, administered dose, dose calibration time, injection time may be entered during acquisition so that these information will be present in the header of the DICOM image. It is, however, strongly recommended to exchange an additional digital scan report form providing all required data, as frequently essential information is still missing. The scan report form should contain study ID, patient randomnisation number, length, weight, gender, administered dose, dose calibration time, injection time, PET scan start time, scan duration, hospital and department, responsible physician and/or address for correspondence. 
Multi-centre QC measures

In order to achieve interchangeability of SUV, a set of PET or PET-CT scanner quality control procedures is required. In case of PET-CT scanners, it is recommended to perform all CT QC measures as recommended and implemented by the manufacturer. Furthermore, other guidelines for execution of diagnostic CT studies and of CT-QC measures on a PET-CT scanner should follow the recommendations provided by the Radiological Society and/or upon indication by a radiologist. In addition, correct alignment between PET and CT data must be checked following manufacturer supplied procedures.

In the remainder of this paper, recommendations focus on PET QC measures specific for accurate SUV measurements. The following PET QC measures are recommended: (1) daily quality control; (2) (cross-)calibration of PET or PET-CT scanner against the (local) dose calibrator used for measurement of patient-specific FDG doses and; (3) an inter-institute cross-calibration and recovery coefficient assessment.

\section{Daily quality control (DailyQC)}

The purpose of a DailyQC is to assess correct functioning of the PET or PET-CT system, i.e., to detect detector failures and/or (overall) drift. Most commercial PET and PET-CT systems are equipped with a (semi-) automatic procedure for performing a DailyQC. It is recommended to follow the procedure provided by the manufacturer. The user has to check whether the DailyQC has passed the test correctly.

2. Quality control of (Cross-)Calibration of PET or PETCT system

The purpose of the (cross-)calibration is to assure correct and direct calibration of the PET or PET-CT camera against the (local) dose calibrator used for measurement of the (FDG) dose to be administered to the patient [32]. In case patientspecific doses are directly ordered from or provided by a pharmaceutical company, the cross-calibration of the PET or PET-CT has to be performed based on a calibrated FDG sample provided by that company. Note that the crosscalibration is the direct relative calibration of the PET system against the (locally) used dose calibrator, and it therefore provides information about potential discrepancies in calibration between PET and dose calibrator. The latter is required because separate calibration of dose calibrators, well counters and PET-CT systems may still result in (small) discrepancies under specific clinical conditions. Standard operating procedures (SOP) for performing the PET (cross) calibration QC are made and provided to all Dutch centres. The procedure has been described elsewhere in more detail [32]. A crucial principle of the calibration QC is that, during execution of this measurement, acquisition and reconstructions settings should be identical to those used during patient studies (and as recommended in this paper). Following the SOP, the cross-calibration factor between the PET or PET-CT scanner and dose calibrator should equal one within $5 \%$.

\section{Image quality and recovery coefficients}

Although the aforementioned cross-calibration QC assures correct calibration for standard conditions, SUV quantification in multi-centre trials and inter-institute interchangeability of SUV is still hampered by differences in applied methodology (e.g., due to use of different scanners, acquisition protocols, reconstruction methods and settings). For example, differences in (reconstructed) image resolution will have a very large effect on SUV quantification in relatively small tumours with volumes up to $10 \mathrm{ml}$ (depending on shape as well). Therefore, it is also required to assess accuracy of SUV using an image quality phantom containing variously sized sphere simulating tumours. Such a phantom will allow assessment of SUV accuracy in 'tumour'-like conditions. The purposes of the 'image quality and recovery coefficients' quality control procedure are: (1) to assess SUV quantification in 'tumour' like structures; (2) to assess correct calibration/ quantification for a nonstandard phantom and; (3) to determine recovery coefficients as function of sphere (tumour) size. The latter data can be used to compensate for differences in image resolution and convergence properties of the available reconstruction algorithm amongst institutes and, if needed, to adapt local acquisition and reconstruction settings such that recovery coefficients match those specified in Tables 5 and 6.

The 'image quality and recovery coefficients' quality control procedure is performed similarly to the "image quality, accuracy of attenuation and scatter corrections" procedure of the NEMA Standards Publication NU 2-2001 "performance measurements of positron emission tomographs". The main difference is that, now, all spheres will be filled with a FDG solution equal to about eight times the activity concentration of the background compartment. Furthermore, acquisition, reconstruction and data analysis (ROI) methods to measure and determine sphere uptake are performed as recommended in this paper for patient studies. A standard operating procedure (SOP "Image Quality and recovery coefficients PET", Dutch version) is available in the Dutch version of the ap-

Table 5 Recovery coefficient (RC) specifications for $\mathrm{VOI}_{\mathrm{A} 50}$

\begin{tabular}{lccc}
\hline Sphere volume $(\mathrm{ml})$ & Expected RC & Maximal RC & Minimal RC \\
\hline 26.52 & 0.77 & 0.83 & 0.71 \\
11.49 & 0.73 & 0.79 & 0.67 \\
5.57 & 0.66 & 0.73 & 0.59 \\
2.57 & 0.60 & 0.68 & 0.53 \\
1.15 & 0.45 & 0.52 & 0.38 \\
0.52 & 0.30 & 0.35 & 0.25 \\
\hline
\end{tabular}


Table 6 Recovery coefficient (RC) specifications for ROI MAX

\begin{tabular}{lccc} 
Sphere volume $(\mathrm{ml})$ & Expected RC & Maximal RC & Minimal RC \\
\hline 26.52 & 0.98 & 1.08 & 0.88 \\
11.49 & 0.95 & 1.05 & 0.85 \\
5.57 & 0.89 & 1.01 & 0.77 \\
2.57 & 0.84 & 0.94 & 0.75 \\
1.15 & 0.63 & 0.74 & 0.51 \\
0.52 & 0.38 & 0.46 & 0.29 \\
\hline
\end{tabular}

proved recommendations protocol. Following the SOP, absolute activity concentration recovery coefficients as function of sphere size are obtained. These recovery coefficients should be within the specifications given in Tables 5 and 6 for the two given ROI (A50\% and MAX).

\section{Limitations, observations and open issues}

The recommendations in this paper aim at standardisation of FDG-PET studies in order to achieve interchangeability of SUV in multi-centre trials. Yet, a few issues are still open for debate and need to be addressed in the near future. Also, during multi-centre phantom measurements, some limitations were encountered and will be discussed below.

First of all, the recommended FDG dose takes into account patient weight, time per bed position, applied bed overlap and scanning mode (2D or 3D). Although patient weight is used directly, the variation in image quality does not depend linearly on patient weight, and the relation between weight, length and gender is ignored. An alternative parameter to vary dose could be measures such as lean body mass, body surface area or body mass index, but their relation with image quality is not resolved as well. Secondly, it is assumed that effects of minimal versus $50 \%$ bed overlap on image SNR equals a factor 2. Similarly, it is also assumed that $2 \mathrm{D}$ and $3 \mathrm{D}$ acquisition mode results in a factor 2 difference of SNR. Ideally, it is optimal to assess image SNR for all scanners using various anthropomorphic phantoms (with various sizes and levels of outside field of view activities) to fully determine differences in image quality amongst various scanners and use those data to specify recommended FDG dose. The recommendations given in this paper, however, offer an advantage over the usually (fixed) dosages applied in most centres, while they are still feasible to be used in a clinical setting. The image quality QC experiments are used to assess any remaining impact of image SNR on SUV quantification. So far, it has been observed that differences between scanners and centres are within $10 \%$ provided recommendations are followed strictly (Fig. 2).

Occasionally, the possibility and flexibility to set acquisition and reconstruction parameters are limited. In practice, however, it was observed that present default reconstruction settings, as implemented by the manufacturer, closely fulfilled the recommendations given in this paper. The limited possibilities to change or overrule the manufacturer-provided settings is a way of standardisation by itself and may therefore be considered as 'beneficial' for the moment. Yet, the impacts of future software and hardware upgrades on SUV quantification need to be carefully monitored. Any change in default acquisition and reconstruction algorithm and their settings will directly have an effect on observed SUV. Any software and hardware upgrade should therefore be clearly specified by the manufacturer, including a description of the effects of the upgrade on image quality (resolution, SNR). The authors therefore hope that PET and PET-CT scanner manufacturers are willing to clearly specify the meaning of acquisition and reconstruction parameters used in their software and graphical user interface. Moreover, sufficient flexibility in changing parameter settings would be beneficial to facilitate matching of image SNR, convergence of iterative reconstruction methods and image resolution in a multicentre studies.

Another observation (data not shown) is that calibration and image quality, especially uniformity of pixel values of reconstructed images, may differ between (same) scanners of the same manufacturer at different sites. The consequence of this observation is that all QC measures have to be performed at each site to verify that the scanner characteristics and specifications fulfill the criteria given in the 'NEDPAS' protocol.

Occasionally, we observed that cross-calibration between PET scanner and dose calibrator revealed a (relative) difference of more than the recommended $5 \%$. The cause of this discrepancy is not fully understood, as the phantom used to calibrate the scanner to the dose calibrator is or can be used to verify correct calibration during the cross-calibration QC measurement. One hypothesis may be that acquisition and reconstruction settings and algorithms used during the calibration procedure are not equal to those during the calibration QC (or verification) measurements and thus not equal to that used during patient studies. Discrepancies may also be caused, e.g., by the use of 'calculated' attenuation correction rather than using a CT-AC or a transmission scan. Other causes might be the use of (slightly) different reconstruction or correction algorithms or settings. Moreover, the calibration must be performed at (a range of) count rates normally encountered in clinical studies. It is therefore of utmost importance that the scanner is calibrated using acquisition and image reconstruction algorithms and settings identical to those used during patient studies. In this way, it is assured that results obtained during calibration are applicable to those obtained during the calibration QC and the patient studies.

An issue to resolve is the use of contrast agents during PETCT studies. Several studies have been performed to assess the effects of intravenous contrast agents on the accuracy and image quality of PET-CT scans [33-39]. Impact of the use of IV contrast on SUV of lesions (tumours and nodes) ranged 
from $0 \%$ to $25 \%$. Amplitude and frequency of observed increase in SUV depend on contrast agent used and specific imaging protocols [40]. Moreover, effects of using intravenous contrast may differ between baseline and posttreatment scans [37]. A simple strategy to avoid any artefact or quantification error is to acquire a dedicated low-dose CT-AC scan prior to PET scanning. After concluding the entire PET procedure, a diagnostic CT scan including use of intravenous contrasts can then be made. Using the latter strategy, it is also feasible to acquire the diagnostic $\mathrm{CT}$ at maximal inspiration to further enhance diagnostic accuracy, while the CT-AC can be used for localisation of FDG avid lesions.

In case of oral contrast agents, this scanning setup cannot be followed to avoid presence of contrast agents in the patient during CT-AC. To date, use of (diluted) oral contrast agents during PET-CT studies is a matter of debate. Various studies have shown that oral contrast agents affect quantification of FDG-PET studies possibly up to $103 \%$ in the case of high density barium contrast [41], but effects on SUV seem to be small when using diluted or other types of contrast agents [42-45]. The amplitude of this effect thus depends strongly on type of contrast agent and a priori unknown local accumulations. Moreover, increase of SUV seemed to be mostly observed in the ascending colon [46]. An interesting alternative might be the use of negative oral contrast agents, which do not affect the accuracy of CT-based attenuation correction, and is therefore a promising possibility for PETCT studies [47, 48]. Use of negative oral contrast will hopefully be of sufficient quality for diagnostic use, which needs to be assessed by the radiology community.

The potential effects of contrast agents on SUV may vary between scans and can create (extra) artificial SUV responses in longitudinal studies. Although effects of $0 \%$ to $25 \%$ seem small, it is just one of the many such (small) factors affecting SUV accuracy and variability. The accumulated resulting inaccuracy led us to argue that all (small) factors contributing to variability in SUV across centres should be controlled as much as possible, and this reasoning is, in fact, the main driver of many of the presented recommendations. Based on phantom experiments (Fig. 2), inter-institute variability due to technical issues could be minimized to within $7 \%(1 \mathrm{SD})$ provided upon strict standardisation of PET procedures. The clinical benefit of using contrast agents should therefore be carefully balanced against potential increased SUV variability and/or incorrect assessments of treatment response up to $10-20 \%$ in some cases $[33,37]$.

\section{Conclusions}

This paper describes a protocol for standardization of quantitative FDG whole body multicentre PET studies. The protocol was successfully introduced in the Netherlands and has been approved by the Netherlands Society of Nuclear Medicine.

Acknowledgements The authors would like to thank the Netherlands Society of Nuclear Medicine and all members of the HOVON imaging working group. In addition, the authors thank all centres participating in the multi-centre study for their participation. Henri Greuter is thanked for providing data on remaining activities in the syringes after administration. Open Access This article is distributed under the terms of the Creative Commons Attribution Noncommercial License which permits any noncommercial use, distribution, and reproduction in any medium, provided the original author(s) and source are credited.

\section{Reference}

1. Fletcher JW, Djulbegovic B, Soares HP, Siegel BA, Lowe VJ, Lyman GH, et al. Recommendations on the use of F-18-FDG PET in oncology. J Nucl Med. 2008;49(3):480-508.

2. Vansteenkiste JF, Stroobants SG. The role of positron emission tomography with $18 \mathrm{~F}$-fluoro-2-deoxy-D-glucose in respiratory oncology. Eur Respir J. 2001;17(4):802-20.

3. Geus-Oei LF, van der Heijden HF, Corstens FH, Oyen WJ. Predictive and prognostic value of FDG-PET in nonsmall-cell lung cancer: a systematic review. Cancer 2007;110(8):1654-64.

4. Weber WA. Use of PET for monitoring cancer therapy and for predicting outcome. J Nucl Med. 2005;46(6):983-95.

5. Avril N, Rose CA, Schelling M, Dose J, Kuhn W, Bense S, et al. Breast imaging with positron emission tomography and fluorine18 fluorodeoxyglucose: use and limitations. J Clin Oncol. 2000;18 (20):3495-502.

6. Bastiaannet E, Groen H, Jager PL, Cobben DCP, van der Graaf WTA, Vaalburg W, et al. The value of FDG-PET in the detection, grading and response to therapy of soft tissue and bone sarcomas; a systematic review and meta-analysis. Cancer Treat Rev. 2004;30 (1):83-101.

7. Borst G, Belderbos J, Boellaard R, Comans E, de Jaeger K, Lammertsma A, et al. Prognostic significance of the 18FDG-PET standardized uptake value for inoperable non-small cell lung cancer patients after high-dose radiotherapy. Lung Cancer 2005;49:S50.

8. Herder GJM, Kramer H, Hoekstra OS, Smit EF, Pruim J, van Tinteren H, et al. Traditional versus up-front [F-18] fluorodeoxyglucose-positron emission tomography staging of non-small-cell lung cancer: a Dutch cooperative randomized study. J Clin Oncol. 2006; 24(12): $1800-6$.

9. Thie JA. Understanding the standardized uptake value, its methods, and implications for usage. J Nucl Med. 2004;45(9):1431-4.

10. Boellaard R, Krak NC, Hoekstra OS, Lammertsma AA. Effects of noise, image resolution, and ROI definition on the accuracy of standard uptake values: a simulation study. J Nucl Med. 2004;45 (9):1519-27.

11. Weber WA. Positron emission tomography as an imaging biomarker. J Clin Oncol. 2006;24(20):3282-92.

12. Westerterp M, Pruim J, Oyen W, Hoekstra O, Paans A, Visser E, et al. Quantification of FDG PET studies using standardised uptake values in multi-centre trials: effects of image reconstruction, resolution and ROI definition parameters. Eur J Nucl Med Mol Imaging. 2007;34 (3):392-404.

13. Freudenberg LS, Rosenbaum-Krumme SJ, Bockisch A, Eberhardt W, Frilling A. Cancer of unknown primary. Recent Results Cancer Res 2008;170:193-202.

14. Weber WA. PET for response assessment in oncology: radiotherapy and chemotherapy. Br J Radiol 2005;78:42-9. 
15. Jaskowiak CJ, Bianco JA, Perlman SB, Fine JP. Influence of reconstruction iterations on F-18-FDG PET/CT standardized uptake values. J Nucl Med. 2005;46(3):424-8.

16. Stahl A, Ott K, Schwaiger M, Weber WA. Comparison of different SUV-based methods for monitoring cytotoxic therapy with FDG PET. Eur J Nucl Med Mol Imaging. 2004;31(11):1471-9.

17. Krak NC, Boellaard R, Hoekstra OS, Twisk JWR, Hoekstra CJ, Lammertsma AA. Effects of ROI definition and reconstruction method on quantitative outcome and applicability in a response monitoring trial. Eur J Nucl Med Mol Imaging. 2005;32(3):294-301.

18. Young H, Baum R, Cremerius U, Herholz K, Hoekstra O, Lammertsma AA, et al. Measurement of clinical and subclinical tumour response using [18F]-fluorodeoxyglucose and positron emission tomography: review and 1999 EORTC recommendations. European Organization for Research and Treatment of Cancer (EORTC) PET Study Group. Eur J Cancer 1999;35(13):1773-82.

19. Shankar LK, Hoffman JM, Bacharach S, Graham MM, Karp J, Lammertsma AA, et al. Consensus recommendations for the use of F-18-FDG PET as an indicator of therapeutic response in patients in national cancer institute trials. J Nucl Med. 2006;47(6):1059-66.

20. Larson SM, Schwartz LH. 18F-FDG PET as a candidate for "qualified biomarker": functional assessment of treatment response in oncology. J Nucl Med 2006;47(6):901-03.

21. Zijlstra JM, Comans EF, van Lingen A, Hoekstra OS, Gundy CM, Coebergh JW, et al. FDG PET in lymphoma: The need for standardization of interpretation. An observer variation study. Nucl Med Common. 2007;28(10):798-803.

22. Juweid ME, Stroobants S, Hoekstra OS, Mottaghy FM, Dietlein M, Guermazi A, et al. Use of positron emission tomography for response assessment of lymphoma: consensus of the Imaging Subcommittee of International Harmonization Project in Lymphoma. J Clin Oncol 2007;25(5):571-8.

23. Coleman RE, Delbeke D, Guiberteau MJ, Conti PS, Royal HD, Weinreb JC, et al. Concurrent PET/CT with an integrated imaging system: intersociety dialogue from the joint working group of the American College of Radiology, the Society of Nuclear Medicine, and the Society of Computed Body Tomography and Magnetic Resonance. J Nucl Med. 2005;46(7):1225-39.

24. Delbeke. Procedure guideline for tumor imaging with F-18-FDG PET/CT 1.0 (vol 47, pg 885, 2006). J Nucl Med. 2006;47(6):903.

25. Watson CC. New, faster, image-based scatter correction for $3 \mathrm{D}$ PET. IEEE Trans Nucl Sci. 2000;47(4):1587-94.

26. Erdi YE, Mawlawi O, Larson SM, Imbriaco M, Yeung H, Finn R, et al. Segmentation of lung lesion volume by adaptive positron emission tomography image thresholding. Cancer 1997;80(12 Suppl):2505-9.

27. Bradley J, Thorstad WL, Mutic S, Miller TR, Dehdashti F, Siegel $\mathrm{BA}$, et al. Impact of FDG-PET on radiation therapy volume delineation in non-small-cell lung cancer. Int J Radiat Oncol Biol Phys 2004;59(1):78-86

28. Giraud P, Grahek D, Montravers F, Carette MF, DeniaudAlexandre E, Julia F, et al. CT and (18)F-deoxyglucose (FDG) image fusion for optimization of conformal radiotherapy of lung cancers. Int J Radiat Oncol Biol Phys 2001;49(5):1249-57.

29. Nestle U, Kremp S, Schaefer-Schuler A, Sebastian-Welsch C, Hellwig D, Rube C, et al. Comparison of different methods for delineation of 18F-FDG PET-positive tissue for target volume definition in radiotherapy of patients with non-small cell lung cancer. J Nucl Med 2005;46(8):1342-8.

30. Black QC, Grills IS, Kestin LL, Wong CYO, Wong JW, Martinez AA, et al. Defining a radiotherapy target with positron emission tomography. Int J Radiat Oncol Biol Phys. 2004;60(4):1272-82.

31. van Dalen JA, Hoffmann AL, Dicken V, Vogel WV, Wiering B, Ruers TJ, et al. A novel iterative method for lesion delineation and volumetric quantification with FDG PET. Nucl Med Commun 2007;28(6):485-93.
32. Greuter HN, Boellaard R, van Lingen A, Franssen EJ, Lammertsma AA. Measurement of 18F-FDG concentrations in blood samples: comparison of direct calibration and standard solution methods. J Nucl Med Technol. 2003;31(4):206-9.

33. An YS, Sheen SS, Oh YJ, Hwang SC, Yoon JK. Nonionic intravenous contrast agent does not cause clinically significant artifacts to F-18-FDG PET/CT in patients with lung cancer. Ann Nucl Med. 2007;21(10):585-92.

34. Antoch G, Freudenberg LS, Stattaus J, Jentzen W, Mueller SP, Debatin JF, et al. Whole-body positron emission tomography-CT: optimized CT using oral and IV contrast materials. AJR Am J Roentgenol 2002;179(6):1555-60.

35. Antoch G, Freudenberg LS, Egelhof T, Stattaus J, Jentzen W, Debatin JF, et al. Focal tracer uptake: a potential artifact in contrastenhanced dual-modality PET/CT scans. J Nucl Med. 2002;43 (10):1339-42.

36. Berthelsen AK, Holm S, Loft A, Klausen TL, Andersen F, Hojgaard L. PET/CT with intravenous contrast can be used for PET attenuation correction in cancer patients. Eur J Nucl Med Mol Imaging 2005;32(10):1167-75.

37. Vera P, Ouvrier MJ, Hapdey S, Thillays M, Pesquet AS, Diologent B, et al. Does chemotherapy influence the quantification of SUV when contrast-enhanced CT is used in PET/CT in lymphoma? Eur J Nucl Med Mol Imaging 2007;34(12):1943-52.

38. Visvikis D, Costa DC, Croasdale I, Lonn AH, Bomanji J, Gacinovic S, et al. CT-based attenuation correction in the calculation of semiquantitative indices of [18F]FDG uptake in PET. Eur J Nucl Med Mol Imaging 2003;30(3):344-53.

39. Yau YY, Chan WS, Tam YM, Vernon P, Wong S, Coel M, et al. Application of intravenous contrast in PET/CT: Does it really introduce significant attenuation correction error? J Nucl Med. 2005;46(2):283-91.

40. Beyer T, Antoch G, Bockisch A, Stattaus J. Optimized intravenous contrast administration for diagnostic whole-body $18 \mathrm{~F}-\mathrm{FDG}$ PET/CT. J Nucl Med 2005;46(3):429-35.

41. Ahmadian A, Ay MR, Bidgoli JH, Sarkar S, Zaidi H. Correction of oral contrast artifacts in CT-based attenuation correction of PET images using an automated segmentation algorithm. Eur J Nucl Med Mol Imaging 2008;5:3542-7.

42. Antoch G, Jentzen W, Freudenberg LS, Stattaus J, Mueller SP, Debatin JF, et al. Effect of oral contrast agents on computed tomography-based positron emission tomography attenuation correction in dual-modality positron emission tomography/computed tomography imaging. Invest Radiol 2003;38(12):784-9.

43. Cohade C, Osman M, Nakamoto Y, Marshall LT, Links JM, Fishman EK, et al. Initial experience with oral contrast in PET/ CT: phantom and clinical studies. J Nucl Med 2003;44(3): 412-6.

44. Dizendorf E, Hany TF, Buck A, von Schulthess GK, Burger C. Cause and magnitude of the error induced by oral CT contrast agent in CT-based attenuation correction of PET emission studies. J Nucl Med 2003;44(5):732-38.

45. Groves AM, Kayani I, Dickson JC, Townsend C, Croasdale I, Syed $\mathrm{R}$, et al. Oral contrast medium in PET/CT: should you or shouldn't you? Eur J Nucl Med Mol Imaging 2005;32(10):1160-6.

46. Dizendorf EV, Treyer V, von Schulthess GK, Hany TF. Application of oral contrast media in coregistered positron emission tomography-CT. AJR Am J Roentgenol 2002;179 (2):477-81.

47. Antoch G, Kuehl H, Kanja J, Lauenstein TC, Schneemann H, Hauth E, et al. Dual-modality PET/CT scanning with negative oral contrast agent to avoid artifacts: introduction and evaluation. Radiology 2004;230(3):879-85.

48. Hausegger K, Reinprecht P, Kau T, Igerc I, Lind P. Clinical experience with a commercially available negative oral contrast medium in PET/CT. Rofo 2005;177(6):796-9. 